\title{
PROPIEDADES PSICOMÉTRICAS Y DIMENSIONALIDAD DE LA VERSIÓN ESPAÑOLA PARA NIÑOS Y ADOLESCENTES DEL FATHER INVOLVEMENT SCALE (FIS) [PSYCHOMETRIC PROPERTIES AND DIMENSIONALITY OF THE SPANISH VERSION OF THE FATHER INVOLVEMENT SCALE (FIS)]
}

\author{
María J. GonZÁlez-Calderón ${ }^{1}$, Miguel Ángel RodríGuez ${ }^{2}$ y Juan Carlos SuÁreZ ${ }^{3}$
}

Cómo referenciar este artículo/How to reference this article:

González-Calderón, M. J., Rodríguez, M. A. y Suárez, J. C. (2014). Propiedades psicométricas y dimensionalidad de la versión española para niños y adolescentes del Father Involvement Scale (FIS) [Psychometric Properties and dimensionality of the Spanish version of the Father Involvement Scale (FIS)]. Acción Psicológica, 11(2), 61-76. http://dx.doi.org/10.5944/ap.11.2.14175

\section{Resumen}

El presente estudio tiene como objetivo evaluar las propiedades psicométricas y dimensionalidad de la versión española para niños y adolescentes del Father Involvement Scale (FIS) en el marco de la IPARTheory. Para ello, se utilizó una muestra de 514 participantes (46.9\% varones) de edades comprendidas entre 9 y 16 años. Además del cuestionario sobre la implicación paterna, los sujetos informaron de su ajuste emocional, el grado interpersonal de poder y prestigio parental y el grado de aceptación y rechazo parental percibidos. Se obtuvieron dos factores de primer orden (implicación expresiva e implicación instrumental) y otro global (implicación paterna). La validez de contenido y criterio, así como la fiabilidad resultan apropiadas para los tres factores. No se encontraron diferencias por sexo.

Palabras claves: Adolescentes; evaluación; implicación paterna; niños; propiedades psicométricas.

\begin{abstract}
The present study aims to evaluate the psychometric properties and dimensionality of the Spanish version for children and adolescents of the Father Involvement Scale (FIS) under the IPARTheory. To do this, a sample of 514 participants (46.9\% male) aged between 9 and 16
\end{abstract}

Agradecimientos: Esta investigación ha sido financiada por el Ministerio de Ciencia e Innovación mediante el proyecto PSI2011-28925

Correspondencia: Profesora Dra. María José González Calderón. Universidad a Distancia de Madrid (Udima). Facultad de CC de la Salud y la Educación. Camino de la Fonda, 20. Collado Villalba (Madrid). CP: 28400. Teléfono: +34 918561694 (Ext. 3502). E-mail: mariajose.gonzalez@udima.es

1 Dpto de Psicología, Facultad de Ciencias de la Salud y la Educación (UDIMA).

2 Dpto de Psicología Evolutiva, Facultad de Ciencias de la Educación (Universidad de Cádiz).

3 Dpto de Metodología de las Ciencias del Comportamiento, Facultad de Psicología (UNED). 
years were used. In addition to the questionnaire on parental involvement subjects reported their emotional adjustment, the degree of parental power and prestige and perceived parental acceptance and rejection. Two first-order factors (expressive and instrumental involvement) and another comprehensive one (parental involvement) were obtained. Content as well as criterion validity of each factor seem to be appropriate and so is their reliability. No sex differences were found in this study.

Key words: Adolescents; assessment; father involvement; children; psychometric properties.

\section{Introducción}

El rol del padre en la educación de los hijos ha evolucionado en los últimos años (Parke, 1995; Veneziano y Rohner, 1998). Tradicionalmente, la figura paterna proporcionaba a la familia, sobre todo, protección y recursos económicos, frente a la materna, que cubría aspectos más emocionales y domésticos (Parsons y Bales, 1955). Sin embargo, resultado de los cambios sociales experimentados desde mediados de los años 70 hasta la actualidad, se ha demandado progresivamente una mayor implicación del padre en aspectos relacionados con el cuidado, la alimentación y la comunicación con los hijos, reservados anteriormente a las madres. De hecho, el tiempo que el padre dedica a los hijos se ha visto incrementado en los últimos años (Pleck y Masciadrelli, 2004).

Se da la circunstancia de que la evolución de las expectativas acerca del "rol del padre» dentro de la familia ha avanzado más rápidamente que los cambios en la conceptualización de éste y el desarrollo de instrumentos que nos permitan evaluar y analizar dicha implicación paterna en el proceso de educación y crianza de los hijos (Finley y Schwartz, 2004). Además, aunque el interés por la contribución paterna en el desarrollo de los hijos y la familia se ha visto incrementado en los últimos años, aún sigue siendo insuficiente (Gryczkowski, Jordan y Mercer, 2010). Se hace necesario, por tanto, incorporar la figura paterna en aquellos estudios que buscan la comprensión del desarrollo y ajuste emocional de los hijos, entre otros aspectos, debido a que constituye una fuente de influencia relevante en la vida de éstos, independientemente de la estructura familiar en cuestión (Arranz y Oliva, 2009; Fields, 2003).

Hasta la fecha, la literatura ha evidenciado una relación positiva entre el grado de implicación paterna y el bienestar de los hijos, y negativa entre el primero y los problemas de conducta filiales (Allgood, Beckert y Peterson, 2012; Amato y Rivera, 1999; Carrasco, Holgado, Rodríguez y del Barrio, 2009; Carlson, 2006) especialmente cuando se trata de hijos vulnerables al desarrollo de problemas comportamentales (Aldous y Mulligan, 2002).

El estudio de la implicación del padre en la familia se ha caracterizado por presentar importantes problemas metodológicos, de entre los que destaca la ausencia de una conceptualización y operativización del significado de dicha «implicación» (Marsiglio, Amato y Day, 2000). Una primera aproximación la conceptualiza como un fenómeno observable y temporal, ya que se limita a identificar el tiempo que el padre pasa con los hijos y a estudiar las interacciones observables entre ambos (Lamb, 1997; Lamb, Pleck y Levine, 1985). Sin embargo, esta perspectiva de la implicación paterna no permite determinar el impacto emocional que el padre posee sobre las vidas de los hijos, posiblemente debido a que el ajuste emocional de éstos está más relacionado con la percepción que los mismos tienen del nivel y calidad de la implicación paterna que con el tiempo que sus padres pasan con ellos (Finley y Schwartz, 2004; Pleck, 1997; Veneziano y Rohner, 1998).

Para superar esta limitación surgen alternativas conceptuales y metodológicas en cuya base está una visión multidimensional de la implicación paterna que incluye componentes afectivos, cognitivos y culturales, así como el análisis de las interacciones observables (Hawkins y Palkovitz, 1999). Desde esta aproximación la implicación paterna se define como el grado en que el padre participa en distintos ámbitos de la vida de sus hijos (Day y Lamb, 2003); entre otros aspectos se encuentran el 
aporte de recursos económicos, los cuidados, la supervisión de las tareas escolares, el fomento de la autonomía, la disciplina y el acompañamiento de los hijos (Bradford, Hawkins, Palkovitz, Christiansen y Day, 2002; Hawkins y Palkovitz, 1999). Estos comportamientos de implicación paternos se vinculan bien a dimensiones «instrumentales» bien a aspectos «expresivos» (Finley y Schwartz, 2004). Las instrumentales incluirían aquellos dominios de la vida del niño relacionados con el aprendizaje de habilidades o contenidos que sirven como instrumentos funcionales para manejarse en su vida (por ejemplo: moral, estudios, disciplina, protección, etc.); en cambio, las dimensiones expresivas estarían relacionadas con la implicación paterna en dominios relativos al desarrollo y la seguridad emocional del hijo (por ejemplo: comportamientos de cuidado, compañerismo, ocio, desarrollo físico, emocional y social).

Esta perspectiva proporciona, fundamentalmente, dos ventajas sobre el modelo de implicación paterna basada en el tiempo compartido: por una parte, permite especificar la presencia del padre en los diferentes ámbitos de la vida de los hijos en los que éste puede participar; por otro lado, permite cuantificar el grado de implicación paterna. Estos aspectos cobran especial relevancia y complejidad cuando se analiza el papel de aquellos padres que no comparten físicamente el mismo hogar del hijo (Finley y Schwartz, 2010).

Basándose en un enfoque multidimensional centrado en el contenido o calidad de las interacciones padre-hijo, Hawkins y Palkovitz (1999) diseñaron un instrumento que medía la implicación paterna, cuya validez contrastaron en un estudio preliminar (Hawkins, Bradford, Christiansen, Palkovitz, Day y Call, 2002). En él los padres valoraban el grado en que estaban implicados en la vida de sus hijos desde el punto de vista tanto afectivo, como cognitivo y conductual, a través de 43 ítems relativos a nueve dimensiones, entre las que se incluían el aporte de recursos, la alabanza, las muestras de afecto, la disciplina o el fomento del éxito del hijo.
No obstante, un aspecto importante a tener en cuenta en este campo de investigación es el hecho de que la información sobre el grado de implicación paterna varía en función de quien proporciona la misma, el propio padre o el hijo. Finley y Schwartz (2004) proponen un enfoque centrado en el niño, que enfatice las percepciones retrospectivas fenomenológicas. Este modelo se ha usado con éxito para estudiar el impacto que posee la percepción de la aceptación y el rechazo paterno en el bienestar emocional de los hijos, así como en su desarrollo psicosocial y sus comportamientos (Rohner, 1986; Rohner y Veneziano, 2001). De acuerdo con este enfoque, lo que más afecta al comportamiento del hijo, tanto el actual como el futuro, es el efecto a largo plazo de la implicación paterna, el cual parece quedar encapsulado en las percepciones retrospectivas de los niños. Asimismo, dicho impacto se considera consecuencia de la percepción del hijo más que de la veracidad de dicha percepción (Finley y Schwartz, 2004). En esta misma línea, han sido numerosos los autores que han subrayado la importancia de la percepción de los hijos acerca de los hábitos de crianza de sus padres (Grusec y Goodnow, 1994; Ivanova e Israel, 2006; Rohner y Veneziano, 2001).

La información obtenida por los hijos, no sólo se ha mostrado fiable (Carrasco, Holgado y del Barrio, 2007; Samper, Cortés, Mestre, Nácher y Tur, 2006) sino también más coherente que la suministrada por sus padres (Gonzales, Cauce y Mason, 1996; Morris, Silk, Steinberg, Sessa, Avenevoli y Essex, 2002). También se ha mostrado válida para estudiar la relación entre la implicación paterna y diversos constructos relativos al ajuste emocional del niño, como el rendimiento escolar, la sintomatología depresiva o los problemas exteriorizados (Paulson, 1994; Roa y del Barrio, 2002). La razón que puede explicar la mayor ventaja del autoinforme es el hecho de que la valoración paterna parece estar sometida a una mayor deseabilidad social que la de los niños (Roa y del Barrio, 2001).

Partiendo del instrumento de implicación paterna elaborado por Hawkins et al. (2002), desde el enfoque multidimensional que se centra en la calidad de las relaciones padres-hijos, 
Finley y Schwartz (2004) crearon otro instrumento, el Father Involvement Scale (FIS) en el que, a diferencia del primero, la fuente de información son los propios hijos. Los autores del mismo asumen, por un lado, una conceptualización multidimensional de la implicación del padre, de modo que se considera que éste puede estar implicado en mayor o menor grado en distintos ámbitos o dimensiones familiares; por otro lado, consideran que lo más relevante no es el tiempo que el padre comparte con sus hijos sino la percepción que tienen éstos del nivel de implicación de la figura parental en los distintos ámbitos de desarrollo de los hijos; y, por último, defienden que el impacto a largo plazo de la implicación paterna sobre los hijos depende de la percepción de los hijos al respecto.

Compartiendo este enfoque y premisas, los objetivos del presente estudio son, por un lado, examinar la dimensionalidad de la versión española del FIS en población infanto-juvenil; y, por otro, analizar sus propiedades psicométricas.

\section{Método}

\section{Participantes}

La muestra estuvo compuesta por 514 niños y adolescentes españoles procedentes de la población general (46.9\% niños y 53.1\% niñas) con edades comprendidas entre los 9 y los 16 años (Media de edad: 13.11; DT: 2.5). La mayoría vivían con sus padres biológicos, los cuales tenían empleos de larga duración cualificados o semicualificados, si bien sólo una minoría de progenitores poseía un título universitario. La práctica mayoría pertenecían al grupo étnico «blanco europeo» y se denominaban católicos.

Los participantes fueron seleccionados por muestreo aleatorio simple de diversos colegios públicos y concertados de diversas ciudades españolas, los cuales también eran mixtos (tenían en sus aulas escolares de ambos sexos). Los criterios de inclusión de la muestra fueron poseer la autorización paterna y presentar el protocolo de evaluación totalmente cumplimentado.

\section{Instrumentos}

Los instrumentos utilizados en el presente estudio consistieron todos en autoinformes cumplimentados por los menores. A continuación, se describen tanto aquel objeto de estudio como los empleados para analizar las propiedades psicométricas del mismo.

\section{Formulario de datos personales}

Se elaboró un formulario ad hoc para recoger información sociodemográfica, como sexo, edad, grupo étnico de pertenencia, religión, estado civil de los padres, progenitores con los que convivían, así como nivel educativo y profesional de éstos, entre otros aspectos.

\section{Adaptación del Father Involvement Scale (FIS; Finley y Schwartz, 2004)}

Es un autoinforme que evalúa el nivel de implicación que los hijos consideran que tienen sus padres en diferentes dominios de su desarrollo (intelectual, emocional, físico, social, etc.). Para ello emplea una escala de 20 ítems, que se corresponden con otros tantos dominios. En el presente estudio se empleó una adaptación ad hoc del FIS en la que cada dominio se acompañó de varios descriptores relacionados con el fin de matizar, explicitar y aclarar el contenido del mismo, así como para mejorar la comprensión de estos. Dichos descriptores no aparecían en la versión original, siendo una inclusión de los autores que han adaptado este instrumento a población española. La pregunta presentada fue siempre la misma, "¿Cuánto se implica mi padre en...?", seguido por el correspondiente dominio, ante el cual se respondía en la siguiente escala tipo Likert: «casi siempre» (4), «bastantes veces» (3), «raras veces» (2) y "casi nunca» (1). Ningún ítem se puntúa a la inversa. La puntuación global se obtiene mediante la suma de cada uno de los 20 ítems, de modo que el rango de puntuaciones posibles oscila de 20 a 80 . Cuanto mayor sea la puntuación en el instrumento, mayor será el grado de implicación percibida 
por el hijo. A diferencia de la escala original de Finley y Schwartz (2004), sólo se presentan ítems relativos a la percepción de los hijos sobre el nivel de implicación de los padres en su desarrollo, no los 20 ítems paralelos acerca de la implicación "deseada» por ellos en otros tantos dominios que empleaban estos autores. Asimismo, las opciones de respuesta tampoco coincidían con los originales, las cuales oscilaban de 1 a 5 .

En el instrumento original, Finley y Schwartz (2004) obtuvieron tres factores: 1) Implicación Expresiva: incluía los ítems relacionados con el cuidado, el compañerismo, el ocio (diversión/jugar), actividades de intercambio/intereses, así como el desarrollo físico, emocional, social y espiritual; 2) Implicación Instrumental: recogía los ítems vinculados a la disciplina, la protección, el desarrollo académico, profesional y moral, los ingresos y el fomento de la independencia y la responsabilidad; y 3) Implicación en el asesoramiento y la guía: el resto de ítems, que en el análisis factorial aparecían incluidos en los dos factores anteriores, conformaron el tercer factor relativo a la tutoría/docencia, el desarrollo de la competencia y el desarrollo intelectual. La consistencia interna de dichos factores, medida con el coeficiente alfa de Cronbach, obtuvo valores de $.93, .91$ y .90 , respectivamente, y la relativa a la puntuación total del instrumento de 97 .

\section{Personality Assessment Questionnaire-} Child version (PAQ; Rohner, 1978, 1990; Rohner y Khaleque, 2005)

El PAQ evalúa el ajuste psicológico autopercibido a través de las percepciones que los individuos tienen de sí mismos en relación a siete dimensiones de personalidad, las cuales reflejan su estado de salud mental: hostilidad/ agresión, dependencia, autoestima negativa, autoeficacia negativa, baja respuesta emocional, inestabilidad emocional y visión del mundo negativa. Sus 42 ítems se responden en una escala tipo Likert de cuatro puntos: "casi siempre verdad» (4), «muchas veces verdad» $(3)$, «a veces verdad» (2) y «casi nunca verdad» (1). Cuanto mayor sea la puntuación, menor ajuste psicológico autopercibido. Una adaptación al español de este instrumento puede consultarse en el presente monográfico.

\section{The Perceived Interpersonal Parental Power and Prestige Questionnaire (3PQ; Rohner; 2011)}

Este instrumento evalúa la percepción que los hijos poseen sobre el poder y el prestigio que consideran que tienen sus padres (frente a sus madres) mediante las siguientes dos dimensiones, cada una constituida por cinco ítems: a) Poder (representa la habilidad del padre o la madre para influir en la opinión y comportamiento de sus hijos); y b) Prestigio (refleja la aprobación social, estima, respeto y admiración hacia el padre o la madre por parte de sus hijos).

Los ítems se contestan en la siguiente escala tipo Likert: «fundamentalmente mi madre» (1), «mi madre más que mi padre» (2), «mi madre y mi padre por igual» (3), «fundamentalmente mi padre» (4) y «mi padre más que mi madre» (5). Por tanto, el rango de puntuaciones por dimensión oscila entre 5 y 25 , donde aquellas inferiores a «15» revelan la percepción de que las madres tienen más poder o prestigio que los padres; puntuaciones por encima de esas cifras indican la tendencia contraria. Una adaptación al español de este instrumento puede consultarse en el presente monográfico.

\section{Parental Acceptance-Rejection Questionnaire-Control; Father and Mother versions (Child PARQ; Rohner, 1990; Rohner y Khaleque, 2005)}

Instrumento autoinformado que evalúa la aceptación y el rechazo, tanto materno como paterno, percibido por los hijos. La mayoría de los ítems se refieren a comportamientos de los padres más que a las actitudes de éstos. En este estudio se empleó tanto la versión abreviada para padres (PARQ-P) como la de madres (PARQ-M), las cuales son idénticas, variando 
únicamente la figura parental sobre cuyas conductas se pregunta. En ambas se examinan las siguientes cuatro dimensiones mediante 29 ítems: cariño-afecto, hostilidad-agresión, indiferencia-negligencia y rechazo indiferenciado. Dichos ítems se puntúan en la siguiente escala Likert: «casi siempre verdad» (4); «muchas veces verdad» (3); "a veces verdad» (2); y casi nunca verdad (1), excepto los que constituyen la dimensión "calidez-afecto» y dos ítems adicionales (16 y 20), cuya valoración es inversa. La suma de los ítems de las cuatro escalas proporciona una medida global de la aceptación y el rechazo percibidos, cuyas puntuaciones oscilan desde 24 (máxima aceptación percibida) hasta 96 (máximo rechazo percibido). Al igual que los anteriores, la adaptación al español de estos cuestionarios puede consultarse en el presente monográfico.

\section{Procedimiento}

En primer lugar se llevó a cabo la traducción de la versión original del FIS, sólo de la escala de implicación percibida, no de la implicación deseada. Posteriormente, se fue perfilando la redacción definitiva de cada uno de los ítems para adaptarlos a la correcta expresión en castellano. Una vez obtenida la versión considerada definitiva por parte de los expertos (véase apéndice A), se procedió a la selección aleatoria de la muestra. Una vez seleccionada ésta, se solicitó la autorización de los distintos centros escolares colaboradores y posterior consentimiento paterno de los alumnos de los grupos participantes. La recogida de datos se llevó a cabo en grupos coincidiendo con los alumnos presentes en cada aula evaluada. La participación de los escolares fue voluntaria, garantizándose el anonimato de sus respuestas. Las instrucciones proporcionadas, así como las condiciones de evaluación, fueron similares en todos los grupos.

Finalizada la recogida de datos se procedió al análisis estadístico de los resultados. Este incluyó, en primer lugar, el estudio de las características descriptivas de la muestra, así como el estudio mediante análisis factoriales confirmatorios (AFC) de primer y segundo or- den de la estructura original del FIS propuesta por Finley y Schwartz (2004). Por último, se analizaron las propiedades psicométricas de la escala y las distintas dimensiones que la constituyen (fiabilidad y validez de criterio) y se obtuvieron los baremos del instrumento separadamente para cada sexo y para cuatro grupos de edad distintos. Los softwares empleados fueron SPSS 15.0 y LISREL.

\section{Resultados}

\section{Examen del ajuste de la versión española del FIS a la estructura original propuesta por Finley y Schwartz (2004)}

De acuerdo con el modelo teórico utilizado para la elaboración del FIS, se propuso una estructura factorial de primer orden, constituida por tres factores. Aunque este modelo ajustaba adecuadamente, un gran número de ítems se solapaban entre los factores confundiendo la delimitación del constructo que cada uno de ellos pretendía evaluar. Por esta razón, se analizó una nueva estructura factorial más parsimoniosa de dos factores: implicación expresiva e implicación instrumental. Este nuevo modelo también mostró un buen ajuste a los datos y además proporcionó un menor solapamiento de los ítems entre factores. Esta estructura bifactorial facilitaba una interpretación más clara y compatible con el constructo teórico, por tanto, se eligió este último modelo como partida para el análisis de la estructura interna del test.

Tras someter el modelo original (modelo 1) a un AFC utilizando mínimos cuadrados no ponderados, se obtuvieron los siguientes índices globales de bondad de ajuste: $\chi_{\text {Satorra-Bentler }}^{2}$ $($ g.l. $=169 ; p=.00)=489.20 ; \operatorname{RMSEA}=.062$ con un intervalo al 90\% comprendido entre .055 y .068; GFI = .98; y AGFI $=.98$.

Con estos resultados se puede afirmar que el modelo propuesto por el autor se ajusta de forma razonable a los datos.

En la figura 1, se muestra la solución completamente estandarizada del modelo 1 . 


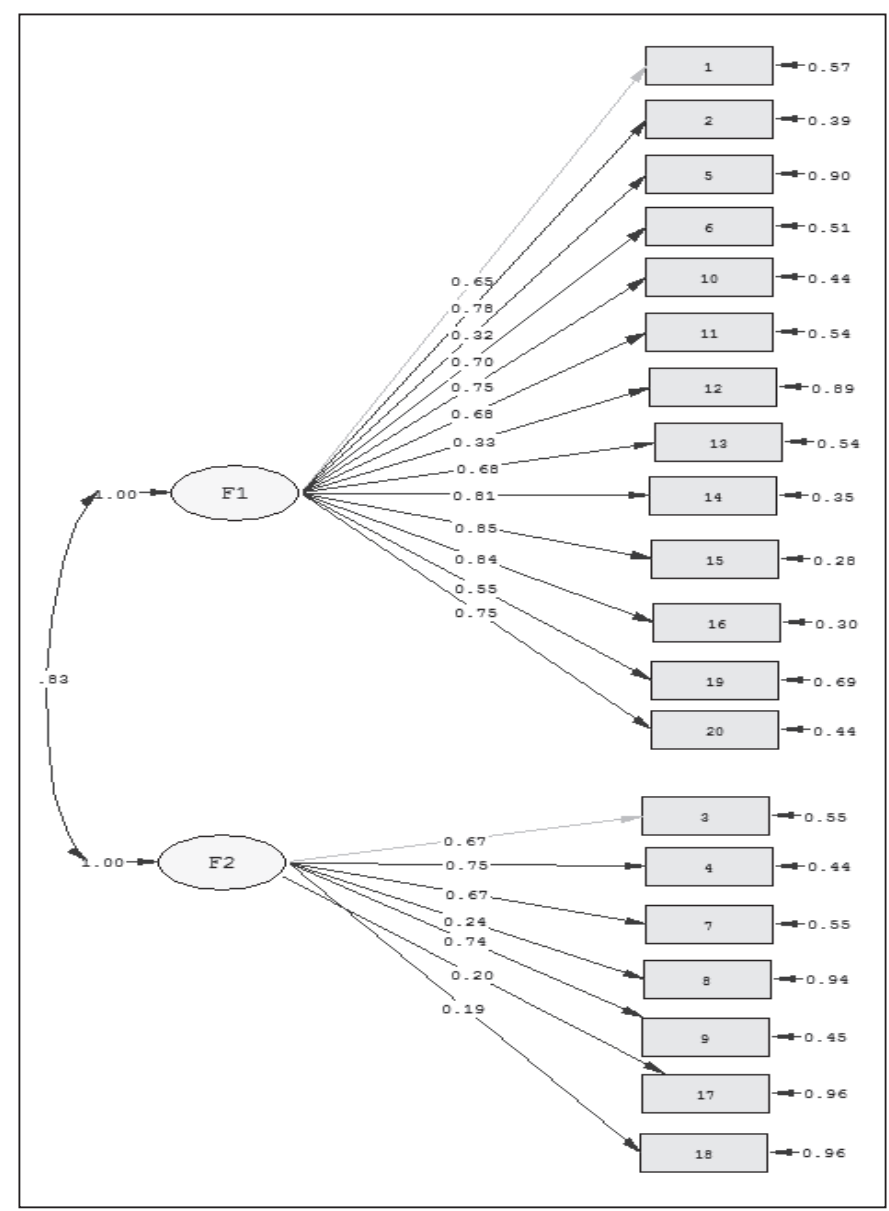

Figura 1. Análisis factorial confirmatorio de primer orden del instrumento FIS. F1: Implicación expresiva; F2: Implicación Instrumental

Dada la elevada correlación entre los distintos factores $(r=.58)$, se estima razonable poner a prueba un segundo modelo (modelo 2) unidimensional que incluya un factor de segundo orden que explique los primeros. Los índices globales de ajuste son: $\chi_{\text {Satorra-Bentler }}^{2}$ (g.l. $=168 ; p=.00)=359.00 ;$ RMSEA $=.048$ con un intervalo al 90\% comprendido entre .041 y .055; GFI = .98; y AGFI =.98.

En la figura 2, se muestra la solución completamente estandarizada del modelo estructural.

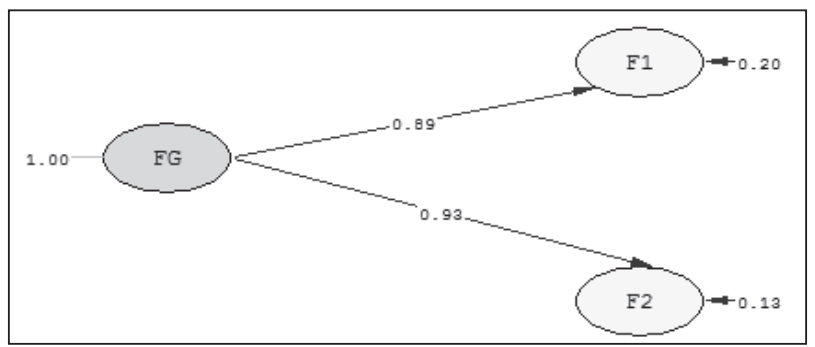

Figura 2. Análisis factorial confirmatorio de segundo orden del instrumento FIS. F1: Implicación expresiva; F2: Implicación Instrumental; FG: Implicación paterna global 
Según estos datos, este segundo modelo mejora el ajuste a los datos, confirmando la existencia de dos factores de primer orden que, a su vez, son explicados por un factor general de orden superior.

Los ítems de la escala Implicación Expresiva según el AFC llevado a cabo se presentan a continuación, junto a la clasificación de dichos ítems en la escala original entre paréntesis: 1 (Asesoramiento y Guía), 2 (Expresivo), 5 (Expresivo), 6 (Expresivo), 10 (Asesoramiento y Guía), 11 (Expresivo), 12 (Instrumental), 13 (Expresivo), 14 (Asesoramiento y Guía), 15 (Expresivo), 16 (Instrumental), 19 (Instrumental) y 20 (Expresivo).

Los ítems de la escala Implicación Instrumental según el AFC llevado a cabo se presentan a continuación: 3 (Expresivo), 4 (Instrumental), 7 (Instrumental), 8 (Instrumental), 9 (Instrumental), 17 (Asesoramiento y Guía) y 18 (Instrumental).

\section{Características psicométricas básicas}

En la tabla 1 se presentan las características psicométricas básicas, tanto de la escala global como de las dos dimensiones que la constituyen según los análisis confirmatorios previamente presentados.

La fiabilidad del instrumento es apropiada para la población española. La consistencia interna evaluada con el coeficiente alfa de Cronbach para las escalas Implicación Expresiva e Implicación Instrumental es de .87 y .62 respectivamente. Asimismo, la consistencia interna de la escala FIS global también es elevada $(\alpha=.88)$. Estos valores se mostraron ligeramente inferiores a los obtenidos por Finley y Schwartz (2004), quienes hallaron valores por encima del .90 en los tres factores que describieron, así como en la escala global.

Por otro lado, el poder de discriminación promedio también es adecuado, tanto el concerniente a la escala entera (.59), como los referentes a cada dimensión individual, cuyos valores oscilan entre .57 y .35 (véase tabla 1) siendo, por tanto, superiores a .30, valor mínimo sugerido por Nunnally y Berstein (1994) para ser considerado adecuado.
Tabla 1.

Propiedades psicométricas de la adaptación del Father Involvement Scale (FIS).

\begin{tabular}{lccr}
\hline \multicolumn{4}{c}{ Dimensiones } \\
\hline & $\begin{array}{c}\text { Implicación } \\
\text { Expresiva }\end{array}$ & $\begin{array}{c}\text { Implicación } \\
\text { Instrumental }\end{array}$ & $\begin{array}{c}\text { FIS } \\
\text { Total }\end{array}$ \\
\hline Media & 39.34 & 20.53 & 59.87 \\
D.T. & 7.60 & 3.84 & 10.31 \\
a & .87 & .62 & .88 \\
D. M. & .57 & .35 & .59 \\
\hline
\end{tabular}

Nota . D.T. = desviación típica; D.M.=discriminación media.

Con el objeto de obtener evidencias de la validez de criterio de la adaptación española del FIS se estudiaron las correlaciones entre las dimensiones del mismo, así como la puntuación global, con las procedentes de otros instrumentos que miden variables estrechamente relacionadas con la implicación paterna, como son la aceptación-rechazo parental, el ajuste psicológico de los hijos, y el poder y prestigio que éstos consideran que tienen sus padres y madres. De acuerdo con lo mostrado en análisis previos, todas estas medidas deberían aparecer vinculadas a los niveles de implicación paterna.

Entre los resultados encontrados puede observarse que a mayor implicación del padre en el desarrollo de los hijos, tanto expresiva como instrumental, menor rechazo percibido por el niño procedente tanto del padre como de la madre, especialmente del primero (véase tabla 2). Asimismo, cuanto mayor es la implicación del padre en el desarrollo del hijo, menores niveles de desajuste psicológico, presenta este último, como también es esperable en base a la literatura al respecto. No obstante, y a diferencia de lo que sucede con la implicación de tipo instrumental y la implicación paterna global percibida, la implicación expresiva no parece relacionarse con el grado de ajuste del sujeto.

Por último, señalar que cuanto más implicado está el padre en la crianza de los hijos, éstos tienden a percibir mayores niveles paternos que maternos tanto de poder como de prestigio dentro del núcleo familiar. 
Tabla 2.

Análisis de la validez de criterio del Father Involvement Scale (FIS).

\begin{tabular}{lcccccc}
\hline \multicolumn{1}{c}{ FIS } & PARQ-P & PARQ-M & 3PQ & Poder & Prestigio & PAQ \\
\hline Implicación expresiva. & $-.74^{* *}$ & $-.33^{* *}$ & .05 & $.32^{* *}$ & $.34^{* *}$ & -.06 \\
Implicación instrumental. & $-.43^{* *}$ & $-.26^{* *}$ & $.21^{* *}$ & $.18^{* *}$ & $.16^{* *}$ & $-.16^{* *}$ \\
FIS (Total). & $-.71^{* *}$ & $-.35^{* *}$ & $.11^{*}$ & $.30^{* *}$ & $.33^{* *}$ & $-.10^{*}$ \\
\hline
\end{tabular}

$* * \mathrm{p}<.01$ (bilateral).

$* \mathrm{p}<.05$ (bilateral).

Nota. FIS (puntuación total del Father Involvement Scale); PARQ-P (puntuación total de la versión para padres del Parental Acceptance-Rejection Questionnaire); PARQ-M (puntuación total de la versión para madres del Parental AcceptanceRejection Questionnaire); 3PQ (puntuación total del instrumento The Perceived Interpersonal Parental Power and Prestige Questionnaire); PODER (puntuación de la escala "Poder" del instrumento The Perceived Interpersonal Parental Power and Prestige Questionnaire; PRESTIGIO (escala "Prestigio" del instrumento The Perceived Interpersonal Parental Power and Prestige Questionnaire; PAQ (Puntuación total de la versión infantil del Personality Assessment Questionnaire-Child versión).

\section{Diferencias por sexo}

De acuerdo con la $t$ de Student para muestras independientes, ninguna de las dimensiones, ni la escala total del instrumen- to, mostraron diferencias por sexo estadísticamente significativas. Las puntuaciones medias y las desviaciones típicas correspondientes para ambos sexos se muestran en la tabla 3.

Tabla 3.

Diferencias por sexo en la escala FIS y sus dimensiones.

\begin{tabular}{lcccc}
\hline & Sexo & Implicación Expresiva & Implicación Expresiva & FIS Total \\
\hline \multirow{2}{*}{ Media } & Chicos. & 39.22 & 20.86 & 60.08 \\
& Chicas. & 39.50 & 20.26 & 59.77 \\
D.T. & Chicos. & 7.62 & 3.85 & 10.48 \\
& Chicas. & 7.51 & $3.78 \mathrm{w}$ & 10.08 \\
& $\mathrm{t}$ & $(\mathrm{t} 537=-0.032, \mathrm{p}=.975)$ & $(\mathrm{t} 535=1.624, \mathrm{p}=.105)$ & $(\mathrm{t} 510=0.342, \mathrm{p}=.732)$ \\
\hline
\end{tabular}

Nota. D.T.= desviación típica.

\section{Baremación del FIS}

En el apéndice B encontramos las puntuaciones directas y percentiles de la adaptación española del instrumento de implicación paterna Father Involvement Scale (FIS) en una muestra de 858 sujetos. Se presentan cinco tablas con información acerca de las puntuaciones en ambas escalas, implicación expresiva e implicación instrumental, así como en la puntuación total del FIS. Las cuatro primeras hacen referencia a sendos rangos de edad (9-10 años; 11-12 años; 13-14 años; y 15-16 años) y la última presenta las puntuaciones directas y los correspondientes percentiles para la muestra de chicos y chicas separadamente.

\section{Discusión}

Este trabajo tiene como principal objetivo examinar la dimensionalidad de la versión española del FIS en población infanto-juvenil; y, para ello, analiza sus propiedades psicométricas. Se pretende que sea una herramienta fiable para estudiar el grado de implicación de la figura paterna en diferentes dominios de la vida de los hijos. 
Para examinar el cuestionario se propuso una estructura bifactorial de primer orden haciendo saturar cada ítem en su dimensión teórica. Dada la elevada correlación obtenida entre los distintos factores, se realizó un análisis factorial confirmatorio de segundo orden que mejoró el ajuste y confirmó la existencia de los factores de primer orden (implicación expresiva e implicación instrumental) subsumidos en un factor general de orden superior denominado implicación paterna. La obtención de dos factores difiere de la estructura original propuesta por Finley y Schwartz (2004), quienes obtuvieron tres dimensiones de primer orden: Implicación Expresiva, Implicación Instrumental e Implicación en el asesoramiento y la guía. No obstante, las diferencias no son muy importantes y se explican fundamentalmente por la naturaleza mixta del tercer factor de asesoramiento y guía de la escala original que obtuvieron Finley y Schwartz (2004). En concreto, los resultados muestran que gran parte de los ítems que componen nuestra escala «implicación expresiva» pertenecen a la escala expresiva original, tres ítems al tercer factor (implicación en el asesoramiento y la guía) y tres a la escala instrumental.

La agrupación de la mayoría de los ítems del tercer componente de la estructura original propuesta por Finley y Schwartz (2004) en nuestro factor expresivo podría sugerir que la implicación paterna en los aspectos intelectuales de los hijos hacen que éstos lo perciban como una expresión de preocupación por ellos más que como una manifestación instrumental o funcional. Lo mismo sucede con el ítem «tareas escolares», teóricamente instrumental, pero que en nuestros datos se agrupa dentro de la escala expresiva. Los otros dos ítems instrumentales que componen esta escala hacen referencia al dinero (que sí parece claramente instrumental) y la protección (de carácter eminentemente expresivo).

En cuanto a los ítems del factor de primer orden que conforman la «implicación instrumental», nuestros resultados son especialmente consistentes con los de la escala original, con la excepción de dos ítems: la implicación en el desarrollo social (ítem 3) y el relativo a aconsejar a los hijos (ítem 17). El primero de ellos podría considerarse tanto expresivo como instrumental, aunque Finley y Schwartz (2004) asumían que era expresivo; asimismo, aconsejar a los hijos suele tener un componente instrumental claro, aunque estos autores lo ubicaban en la categoría mixta.

Por todo ello, en términos generales, si comparamos la estructura factorial extraída de nuestro análisis con la obtenida en la escala original, puede afirmarse que la adaptación española es compatible teóricamente con la estructura original propuesta. Estas similitudes junto con la asociación significativa hallada entre las dimensiones del instrumento y variables estrechamente relacionadas con la implicación paterna, como son la aceptación y el rechazo paterno, el ajuste psicológico de los hijos, y el poder y prestigio interpersonal asignado a los padres, muestran adecuadas evidencias de validez de constructo a la estructura propuesta.

Entre los resultados encontrados puede observarse que, de acuerdo con la literatura, a mayor implicación del padre en el desarrollo de los hijos, tanto expresiva como instrumental, mejor ajuste psicológico de éstos (Aldous y Mulligan, 2002; Allgood et al., 2012; Amato y Rivera, 1999; Carrasco et al., 2009; Carlson, 2006; Flouri y Buchanan, 2003; Rohner, 1998; Veneziano, 2000) y menor rechazo tanto materno como paterno percibido por los hijos (Finley y Schwartz, 2008); asimismo, también se han hallado vínculos con mayores niveles de poder y prestigio asignado a los padres, como se esperaba. Por tanto, el conjunto de estos datos no sólo está en sintonía con los resultados obtenidos en el marco de la IPARTheory (Rohner, 1986; Rohner y Veneziano, 2001), sino que son acordes a la literatura que evidencia una relación positiva entre el grado de implicación paterna y el bienestar de los hijos, o la falta de dicha implicación y la presencia de problemas de conducta en los hijos (Allgood et al., 2012; Amato y Rivera, 1999; Carrasco et al., 2009; Carlson, 2006).

La fiabilidad del instrumento también resulta apropiada para la población española, ya que los datos avalan la fiabilidad de ambas escalas, implicación expresiva e implicación ins- 
trumental, así como de la escala total. Nuestros resultados se aproximan mucho a los que obtuvieron Finley y Schwartz, si bien éstos, como hemos mencionado, utilizaron tres factores, además de la puntuación global. Por otro lado, el poder de discriminación promedio también resulta adecuado, tanto el concerniente a la escala entera, como los referentes a cada dimensión separadamente.

Para ninguna de las dimensiones, ni para la escala total, se encontraron diferencias entre los niños y las niñas en el FIS. Por ello, los baremos para el cuestionario aparecen por grupos de edades en relación a la puntuación total y a los factores implicación expresiva e implicación instrumental.

Creemos que los hallazgos de este trabajo van a permitir en el futuro estudiar en población española la importancia de la implicación del padre en los diferentes ámbitos en los que el desarrollo de los hijos tiene lugar. Este cuestionario posibilitará establecer relaciones entre la implicación, la aceptación y el rechazo paternos, así como entre aquellos y el grado de poder y prestigio atribuidos en el seno familiar. Esto, sin duda, puede suponer un impulso en el desarrollo de los conceptos y postulados de la IPARTheory. Además, el estudio de la implicación del padre en la vida de los hijos no sólo despierta el interés en el marco de dicha teoría sino que es un aspecto determinante en muchas aproximaciones teóricas y ámbitos de estudio e intervención como son los psicológicos, los judiciales y los sociales, para los que puede ser una herramienta de gran utilidad.

En cuanto a las limitaciones del estudio, el empleo de diferentes fuentes o informantes además de contar con los datos autoinformados hubiera permitido un mejor análisis de las percepciones tanto de la implicación paterna como del ajuste psicológico de los hijos. No obstante, como se ha descrito previamente, el FIS ha mostrado adecuados niveles de fiabilidad y validez, lo que sustenta los resultados hallados. Futuros estudios deberán emplear distintas fuentes de información, analizar la estabilidad temporal del instrumento y examinar la invarianza factorial por grupos de edad y sexo.

\section{Apéndice A.}

Ítems de la adaptación española del Father Involvement Scale (FIS) y dimensiones a las que pertenecen.

\begin{tabular}{cll}
\hline Ítem & \multicolumn{1}{c}{ Descripción: ¿Cuánto se implica mi padre en.. ? } & \multicolumn{1}{c}{ Dimensión } \\
\hline 1 & $\begin{array}{l}\text { Mi desarrollo intelectual (Ejemplo: me insiste en que le pregunte lo que no } \\
\text { entienda). }\end{array}$ & Implicación Expresiva. \\
2 & Mi desarrollo emocional (Ejemplo: se preocupa por saber cómo me siento). \\
3 & Mi desarrollo social (Ejemplo: me pregunta por mis amigos o compañeros). \\
4 & $\begin{array}{l}\text { Mi desarrollo Ético/moral (Ejemplo: me hace notar la diferencia entre lo que } \\
\text { está bien y está mal). }\end{array}$ & $\begin{array}{l}\text { Implicación Expresiva. } \\
\text { Implicación Instrumental. }\end{array}$ \\
5 & Mi desarrollo espiritual (Ejemplo: me habla de religión, me lleva a misa). \\
6 & $\begin{array}{l}\text { Mi desarrollo físico (Ejemplo: me cuida cuando estoy malo/ me lleva al } \\
\text { médico). }\end{array}$ & Implicación Expresiva. \\
7 & $\begin{array}{l}\text { Mi desarrollo profesional (Ejemplo: se interesa por lo que quiero ser de } \\
\text { mayor). }\end{array}$ & Implicación Instrumental. \\
8 & $\begin{array}{l}\text { Mi desarrollo de la responsabilidad (Ejemplo: me recrimina cuando no hago } \\
\text { algo a lo que me comprometí). }\end{array}$ & Implicación Instrumental. \\
9 & $\begin{array}{l}\text { Mi desarrollo de la independencia (Ejemplo: me anima a que haga cosas por } \\
\text { mi mismo). }\end{array}$ & Implicación Instrumental. \\
10 & $\begin{array}{l}\text { Mi desarrollo de la competencia (Ejemplo: me ayuda a hacer cosas nuevas o o } \\
\text { que son difíciles para mí ). }\end{array}$ & Implicación Expresiva.
\end{tabular}




\begin{tabular}{|c|c|c|}
\hline Ítem & Descripción: ¿Cuánto se implica mi padre en.. ? & Dimensión \\
\hline 11 & Mi ocio, diversión, juego (Ejemplo: ir al cine, parque, partido, deporte). & Implicación Expresiva. \\
\hline 12 & Asignarme dinero (Ejemplo: me da dinero, la paga ...). & Implicación Expresiva. \\
\hline 13 & $\begin{array}{l}\text { Actividades e intereses compartidos (Ejemplo: televisión, música, deporte, } \\
\text { etc.). }\end{array}$ & Implicación Expresiva. \\
\hline 14 & $\begin{array}{l}\text { Orientarme (Ejemplo: me sirve de guía y me indica los pasos a seguir para } \\
\text { realizar una tarea). }\end{array}$ & Implicación Expresiva. \\
\hline 15 & Quererme (Ejemplo: me hace sentir querido). & Implicación Expresiva. \\
\hline 16 & Protegerme (Ejemplo: me hace sentir protegido). & Implicación Expresiva. \\
\hline 17 & Aconsejarme (Ejemplo: me da consejos). & Implicación Instrumental. \\
\hline 18 & Castigarme (Ejemplo: me castiga cuando hago algo mal). & Implicación Instrumental. \\
\hline 19 & Mis trabajos y tareas escolares (Ejemplo: me ayuda a hacer los deberes). & Implicación Expresiva. \\
\hline 20 & Acompañarme (Ejemplo: pasamos tiempo juntos). & Implicación Expresiva. \\
\hline
\end{tabular}

Apéndice B.

Baremos del Father Involvement Scale (FIS).

Baremos por edad del Father Involvement Scale (FIS) para 9-10 años

\begin{tabular}{ccccc}
\hline Pc & \multicolumn{3}{c}{ Puntuaciones directas } & \multirow{2}{*}{ Pc } \\
\cline { 2 - 4 } & $\begin{array}{c}\text { Implicación } \\
\text { Expresiva }\end{array}$ & $\begin{array}{c}\text { Implicación } \\
\text { Instrumental }\end{array}$ & $\begin{array}{c}\text { Implicación } \\
\text { Total }\end{array}$ & \\
\hline 1 & $13-19$ & 7 & $20-30$ & 1 \\
25 & $20-37$ & $8-18$ & $31-57$ & 25 \\
50 & $38-42$ & $19-20$ & $58-61$ & 50 \\
75 & $43-46$ & $21-23$ & $62-67$ & 75 \\
100 & $47-52$ & $24-28$ & $68-80$ & 100 \\
\hline N & 109 & 109 & 109 & N \\
Media & 40.84 & 19.95 & 60.80 & Media \\
D. T. & 6.22 & 3.79 & 8.78 & D. T. \\
\hline
\end{tabular}

Nota. D.T. = desviación típica.

Baremos por edad del Father Involvement Scale (FIS) para 11-12 años

\begin{tabular}{ccccc}
\hline Pc & \multicolumn{3}{c}{ Puntuaciones directas } & \multirow{2}{*}{ Pc } \\
\cline { 2 - 4 } & $\begin{array}{c}\text { Implicación } \\
\text { Expresiva }\end{array}$ & $\begin{array}{c}\text { Implicación } \\
\text { Instrumental }\end{array}$ & $\begin{array}{c}\text { Implicación } \\
\text { Total }\end{array}$ & 1 \\
\hline 1 & $13-15$ & $7-11$ & $20-26$ & 25 \\
25 & $16-38$ & $12-19$ & $27-57$ & 50 \\
50 & $39-42$ & $20-22$ & $58-64$ & 75 \\
75 & $43-45$ & $23-24$ & $65-68$ & 100 \\
100 & $46-52$ & $25-28$ & $69-80$ & N \\
N & 279 & 279 & 279 & Media \\
Media & 40.26 & 21.18 & 61.44 & D. T. \\
D. T. & $13-15$ & $7-11$ & $20-26$ & \\
\hline
\end{tabular}

Nota. D.T. = desviación típica. 
Baremos por edad del Father Involvement Scale (FIS) para 13-14 años

\begin{tabular}{ccccc}
\hline Pc & \multicolumn{3}{c}{ Puntuaciones directas } & Pc \\
\cline { 2 - 4 } & $\begin{array}{c}\text { Implicación } \\
\text { Expresiva }\end{array}$ & $\begin{array}{c}\text { Implicación } \\
\text { Instrumental }\end{array}$ & $\begin{array}{c}\text { Implicación } \\
\text { Total }\end{array}$ & 1 \\
\hline 1 & 13 & 7 & $20-21$ & 25 \\
25 & $14-36$ & $8-19$ & $22-56$ & 50 \\
50 & $37-40$ & $20-22$ & $57-62$ & 75 \\
75 & $41-43$ & $23-24$ & $63-67$ & 100 \\
100 & $44-52$ & $25-28$ & $68-80$ & $\mathrm{~N}$ \\
N & 310 & 310 & 310 & Media \\
Media & 38.69 & 21.24 & 59.93 & D. T. \\
D. T. & 7.73 & 3.97 & 10.85 & \\
\hline
\end{tabular}

Nota. D.T. = desviación típica.

Baremos por edad del Father Involvement Scale (FIS) para 15-16 años

\begin{tabular}{ccccc}
\hline Pc & \multicolumn{3}{c}{ Puntuaciones directas } & Pc \\
\cline { 2 - 4 } & $\begin{array}{c}\text { Implicación } \\
\text { Expresiva }\end{array}$ & $\begin{array}{c}\text { Implicación } \\
\text { Instrumental }\end{array}$ & $\begin{array}{c}\text { Implicación } \\
\text { Total }\end{array}$ & $20-27$ \\
25 & $13-16$ & $7-9$ & $28-54$ & 25 \\
50 & $17-34$ & $10-19$ & $55-60$ & 50 \\
75 & $35-39$ & $20-21$ & $61-65$ & 75 \\
100 & $40-43$ & $22-23$ & $66-80$ & 100 \\
\hline N & $44-52$ & $24-28$ & 160 & N \\
Media & 160 & 160 & 20.89 & Media \\
D. T. & 58.66 & 37.77 & 9.78 & D. T. \\
\hline
\end{tabular}

Nota. D.T. = desviación típica.

Baremos por sexo del Father Involvement Scale (FIS) para la muestra total Baremos por sexo del Child-PARQ-P para la muestra total

\begin{tabular}{ccccc}
\hline \multirow{2}{*}{ Pc } & \multicolumn{3}{c}{ Puntuaciones directas } & \multirow{2}{*}{ Pc } \\
\cline { 2 - 4 } & Chicos & Chicas & Total & 1 \\
1 & $20-22$ & $20-26$ & $20-25$ & 10 \\
10 & $23-49$ & $27-46$ & $26-47$ & 20 \\
20 & $50-54$ & $47-54$ & $48-54$ & 30 \\
30 & $55-57$ & $55-57$ & $55-57$ & 40 \\
40 & $58-60$ & $58-60$ & $58-60$ & 50 \\
50 & $61-62$ & $61-62$ & $61-62$ & 60 \\
60 & $63-64$ & $63-64$ & $63-64$ & 70 \\
70 & $65-66$ & $65-66$ & $65-66$ & 80 \\
80 & $67-68$ & $67-68$ & $67-68$ & 90 \\
90 & $69-71$ & $69-71$ & $69-71$ & 100 \\
100 & $72-80$ & $72-80$ & $72-80$ & \\
\hline
\end{tabular}




\begin{tabular}{ccccc}
\hline \multirow{2}{*}{ Pc } & \multicolumn{3}{c}{ Puntuaciones directas } & \multirow{2}{*}{ Pc } \\
\cline { 2 - 4 } & Chicos & Chicas & Total & N \\
N & 381 & 475 & 858 & Media \\
Media & 60.45 & 60.22 & 60.29 & D. T. \\
D. T. & 9.84 & 10.20 & 10.07 & \\
\hline
\end{tabular}

Nota. D.T. = desviación típica; Diferencia de medias $\left(\mathrm{t}_{854}=0.326, \mathrm{p}=.744\right)$.

\section{Referencias}

Achenbach, T. y Rescorla, L. (2001). The Manual for the ASEBA School-Age Forms \& Profiles. Burlington: University of Vermont, Research Center for Children, Youth, and Families.

Aldous, J. y Mulligan, G. M. (2002). Fathers' child care and children's behavior problems: A longitudinal study. Journal of Family Issues, 23, 624-647.

Allgood, S. M., Beckert, T. E. y Peterson, C. (2012). The role of father involvement in

the perceived psychological well-being of young adult daughters: A retrospective study. North American Journal of Psychology, 14, 95-110.

Amato, P. R. y Rivera, F. (1999). Paternal involvement and children's behavior problems. Journal of Marriage and the Family, 61, 295-313. doi: $10.2307 / 353755$

Arranz, E. y Oliva, A. (2009). Desarrollo psicológico en las nuevas estructuras familiares (pp 15-34). Madrid: Pirámide.

Bradford, K. P., Hawkins, A., Palkovitz, R., Christiansen, S. L. y Day, R. D. (2002). The inventory of father involvement: A pilot study of a new measure of father involvement. Journal of Men's Studies, 10, 183.

Carlson, M. J. (2006). Family structure, father involvement, and adolescent behavior outcomes. Journal of Marriage and the Family, 68, 137-154. doi: 10.1111/j.1741-3737.2006.00239.x

Carrasco, M. A., Holgado, P. y del Barrio M. V. (2007). Análisis de la estructura del cuestionario de comportamiento parental para niños (CRPBI) en población española. Revista Iberoamericana de Diagnóstico y Evaluación Psicológica, 24, 95-120.
Carrasco, M. A., Holgado, P., Rodríguez, M. A. y del Barrio M. V. (2007). Concurrent and across-time relations between mother-father hostility and children's aggression: a longitudinal study. Journal of family violence, 24, 213-220. doi: 10.1007/ s10896-009-9222-y

Day, R. D. y Lamb, M. E. (Eds.). (2003). Conceptualizing and measuring father involvement. Mahwah, NJ: Lawrence Erlbaum Associates.

Fields, J. (2003). Children's living arrangements and characteristics: March 2002. Current Population Reports, 20-547. Washington, DC: U. S. Census Bureau.

Finley, G. E. y Schwartz, S. J. (2004). The father involvement and nurturant fathering scales: Retrospective measures for adolescent and adult children. Educational and Psychological Measurement, 64, 143-164. doi: 10.1177/0013164403258453

Finley, G. E. y Schwartz, S. J. (2008). Perceived Paternal and Maternal Involvement: Factor Structures, Mean Differences, and Parental Roles. Fathering, 6, 62-82.

Finley, G. E. y Schwartz, S. J. (2010). The divided world of the child: Divorce and long-term psychosocial adjustment. Family Court Review, 48, 516-527. doi: 10.1111/j.1744-1617.2010.01326.x

Flouri, E. y Buchanan, A. (2003). The role of father involvement and mother Involvement in adolescents' psychological well-being. British Journal of Social Work, 33, 399-406.

Gonzales, N. A., Cauce, A. M. y Mason, C. A. (1996). Interobserver agreement in the assessment of parental behavior and parent-adolescent conflict: African American mothers, daughters, and independent observers. Child Development, 67, 1483-1498. doi: 10.1111/j.1467-8624.1996. tb01809.x 
Grusec, J. y Goodnow, J. (1994). Impact of parental discipline methods on the child's internalization of values: A reconceptualization of current points of view. Developmental Psychology, 30, 4-19. doi: 10.1037/0012-1649.30.1.4

Gryczkowski, M., Jordan, S. y Mercer, S. (2010). Differential relations between mothers' and fathers' parenting practices and child externalizing behavior. Journal of child and Family studies, 19, 539-546.

Hawkins, A. J., Bradford, K. P., Christiansen, S. L., Palkovitz, R., Day, R. D. y Call, R. A. (2002). The Inventory of Father Involvement: A pilot study of a new measure of father involvement. Journal of Men's Studies, 10, 183-196. doi: 10.3149/ jms.1002.183

Hawkins, A. J. y Palkovitz, R. (1999). Beyond ticks and clicks: The need for more diverse and broader conceptualizations and measures of father involvement. Journal of Men's Studies, 8, 11-32. doi: 10.3149/jms.0801.11

Ivanova, M. e Israel, A. (2006). Family stability as a protective factor against psychopathology for urban children receiving psychological services. Journal of Clinical Child and Adolescent Psychology, 35, 564-570. doi: 10.1207/s15374424jccp3504_7

Lamb, M. E. (Ed.). (1997). The role of the father in child development. Hoboken, NJ: JohnWiley.

Lamb, M. E., Pleck, J. H. y Levine, J. A. (1985). The role of the father in child development: The effects of increased paternal involvement. En B. Lahey y A. Kazdin (Eds.), Advances in clinical child psychology, vol. 8 (pp. 229-266). New York: Plenum. doi: 10.1007/978-1-4613-9820-2_7

Marsiglio, W., Amato, P. y Day, R. D. (2000). Scholarship on fatherhood in the 1990s and beyond. Journal of Marriage and the Family, 62, 11731191. doi: 10.1111/j.1741-3737.2000.01173.x

Morris, A., Silk, J., Steinberg, L., Sessa, F., Avenevoli, S. y Essex, M. (2002). Temperamental vulnerability and negative parenting as interacting of child adjustment. Journal of Marriage and Family, 64, 461-471. doi: 10.1111/j.17413737.2002.00461.x

Nunnally, J. C. y Berstein, I. H. (1994). Psychometric theory (3. ${ }^{\text {a }}$ ed). New York: McGraw-Hill.
Parke, R. D. (1995). Fathers and families. En M. H. Bornstein (Ed.), Handbook of parenting, vol. 3. Status and social conditions of parenting (pp. 2763). Hillsdale, NJ: Lawrence Erlbaum.

Parsons, T. y Bales, R. F. (1955). Family, socialization, and interaction process. Glencoe, IL: Free Press.

Paulson, S. (1994). Relations of parenting style and parental involvement with ninth-grade students' achievement. Journal of Early Adolescence, 14, 250-267. doi: 10.1177/027243169401400208

Pleck, J. H. (1997). Paternal involvement: levels, sources, and consequences. En M. E. Lamb (Ed.), The role of the father in child development (pp. 66-103). Hoboken, NJ: John Wiley.

Pleck, J. H. y Masciadrelli, B. P. (2004). Paternal involvement by U. S. residential fathers: levels, sources, and consequences. En M. E. Lamb (Ed.), The role of the father in child development, (pp. 222-271). Hoboken, NJ: Wiley.

Roa, L. y del Barrio, V. (2001). Adaptación del cuestionario de crianza parental (PCRI-A Gerard, 1994) a la población española. Revista Latinoamericana de Psicología, 33, 329-341.

Roa, L. y del Barrio, V. (2002). Cuestionario de percepción de crianza para niños y adolescentes. Psicología Educativa, 8, 37-51.

Rohner, R. P. (1978). Development and validation of the Personality Assessment Questionnaire: Test Manual. Beverly Hills, CA: Sage Publications, Inc

Rohner, R. P. (1986). The warmth dimension: Foundations of parental acceptance-rejection theory. Beverly Hills, CA: Sage Publications, Inc.

Rohner, R. P. (1990). Handbook for the study of parental acceptance and rejection (3. $\left.{ }^{a} e d\right)$. Storrs, CT: Rohner research publications.

Rohner, R. P. (1998). Father love and child development: History and current evidence. Current Directions in Psychological Science, 7, 157-161.

Rohner, R. P. (2011). Parental Power-Prestige Questionnaire (3PQ). En R. P. Rohner, Measures for use in parental acceptance-rejection research (pp 134135). Storrs, CT: Rohner research publications.

Rohner, R. P. y Khaleque, A. (2005). Personality Assessment Questionnaire (PAQ): Test manual. En R. P. Rohner y A. Khaleque (Eds), Handbook for the 
study of parental acceptance and rejection $\left(4 .^{\mathrm{a}} \mathrm{ed}\right)$ (pp 187-225). Storrs. CT: Rohner Research Pub.

Rohner, R. P. y Veneziano, R. A. (2001). The importance of father love: History and contemporary evidence. Review of General Psychology, 5, 382405. doi: 10.1037/1089-2680.5.4.382.

Samper, P., Cortés, M., Mestre, V., Nácher, M. y Tur, A. (2006). Adaptación del Child's Report of Parent Behavior Inventory (Schaefer, 1965) a población española. Psicothema, 18, 263-271.
Veneziano, R. A. (2000). Perceived paternal and maternal acceptance and rural African American and European American youths' psychological adjustment. Journal of Marriage and the Family, 62, 123-132.

Veneziano, R. A. y Rohner, R. P. (1998). Perceived paternal acceptance, paternal involvement, and youths' psychological adjustment in a rural, biracial southern community. Journal of Marriage and the Family, 60, 335-343. 